\title{
Um Curso de Direito Internacional Ambiental: Elementos e Objetivos
}

\section{Catherine Tinker ${ }^{1}$}

\section{INTRODUÇÃ $\mathrm{O}^{2}$}

Este ensaio tem por objetivo descrever alguns dos elementos do curso de pósgraduação em Direito na disciplina Direito Internacional Ambiental (DIA). Nesse sentido, o termo $D I A$ foi usado para enfatizar que o campo é baseado principalmente no Direito Internacional, com visões adicionais de direito, política e ecologia ambientais. O termo também foi usado para enfatizar que é um estudo de peso, profundo e abrangente, que requer preparação, estudo, dedicação em direito internacional sobre o assunto, e não só um estudo

1 A autora faz uma homenagem à sua mãe, MILDRED HELEN GALLMEIER TINKER, nascida em St. Louis, Missouri, em 5 de abril de 1915: "Minha mãe trabalhou como bibliotecária na Universidade no Estado do Iowa por muitos anos. Após aposentar-se mudou-se para a cidade de Nova Iorque, onde mora e trabalha há mais de 10 anos, parte da semana, na biblioteca de uma ONG da ONU, o Conselbo de População (Population Council), um centro de pesquisas. Ela chegou comigo ao Brasil em agosto de 2004, e desde então participa ativamente da minha experiência de Professora Visitante do CAPES, na UFRGS Faculdade de Direito. Apesar da idade, está sempre pronta para os eventos e festas do grupo, mesmo sem falar nenhuma palavra em português. Contudo, está sempre bem espiritualmente, sempre gentil e graciosa. Eu agredeço a ela por todo o seu amor e dedicação e por estar comigo aqui em Porto Alegre, participando de um momento tão peculiar em minha vida profissional".

- A autora deseja, também, agradecer à Professora Doutora Cláudia Lima Marques, Professora Titular de Direito Internacional Privado da Faculdade de Direito da UFRGS e Vice-Coordenadora do Programa de Pós-Graduação em Direito, pelo convite para lecionar no Programa e por sua grande amizade, parceria e assistência. Agradece também à CAPES pela generosa bolsa como Professora Visitante Estrangeira na UFRGS e pela oportunidade de fazer parte deste excepcional Programa de PósGraduação em Direito da Faculdade de Direito da UFRGS. Do mesmo modo, agradece aos estudantes da Faculdade de Direito, especialmente aqueles envolvidos no Programa de Intercâmbio CAPESFIPSE (Brasil-EUA) da UFRGS e da Universidade Federal do Pará, e aos estudantes de outros países, pela experiência viva de sala de aula e do intercâmbio de pesquisa e pela excelência de suas participações 
comparativo das leis e políticas ambientais nacionais. Obviamente, as idéias de uma análise comparativa das leis ambientais nacionais podem ser úteis na compreensão da questão, na procura de bons modelos ou melhores práticas, ou na apreciação das diferenças culturais e das necessidades do direito civil, do direito comum ou de outros sistemas legais.

Fundamentalmente, no entanto, o $D I A \operatorname{diz}$ respeito àqueles instrumentos e documentos do Direito Internacional sobre o meio ambiente e o desenvolvimento sustentável, incluindo acordos, direito costumeiro, princípios gerais de direito e declarações de soft law oriundas das reuniões ou congressos internacionais, além de escritos de juristas qualificados de várias regiões do mundo.

Na exploração do desenvolvimento da disciplina de $D I A$, especialmente a partir dos anos 70 até os dias de hoje, há referência contínua ao trabalho das Nações Unidas e agências especializadas da ONU, onde muito do novo direito internacional ambiental foi criado e interpretado e no qual atores da sociedade civil têm um papel significante, ao lado dos governos e organizações intergovernamentais, em projetos de lei, no trabalho conformidade de interpretação e monitoração com o $D I A$ nos Estados membros da ONU, freqüentemente em parceria com governos e setores privados.

A complexidade das questões em suas dimensões econômicas, sociais e ambientais e os efeitos de gtandes proporções das atividades humanas presentes, assim como a incerteza científica, fazem as decisões em relação à política e ao direito serem ainda mais desafiadoras. Ao mesmo tempo, o reconhecimento do efeito global das atividades em qualquer parte do planeta e a incapacidade de qualquer governo para enfocar danos ou problemas ambientais internacionais levam à conclusão inevitável de que a cooperação regional e internacional é um imperativo. $O$ verdadeiro desafio do $D I A$ é descobrir interdisciplinariamente o nivel necessário de cooperação diante dos desentendimentos políticos, da falta de financiamerito adequado para programas e do desentendimento sobre os métodos e resultados desejáveis - na verdade, geralmente baseados em diferenças de valores, da análise de custo-benefício e do conselho científico.

e interesses, em particular a Antonia Espíndola Longioni Klee, Thomaz Francisco Silveira de Araújo Santos, Rafael Pellegrini Ribeiro, Maitê de Souza Schmitz, Laura Martins Miller, Miguel Kreling, Simone Regina Backes, Raquel Thais Hunsche, Fernando Lusa Bordin, Daniel Caye, Rafael Balestrin e muitos outros, incluindo todos aqueles alunos do Programa de Pós-Graduação em Direito da UFRGS que atenderam às aulas de Direito Internacional Ambiental e o Direito Internacional Privado no segundo semestre de 2004 e no primeiro semestre de 2005, e, especialmente, àqueles que contribuíram com artigos pra este número dos Cadernos de Pós-Graduação: os doutorados Bibiana Graeff Chagas Pinto, Eugênio Battalli e Fernando Linhares, e os mestrandos Raul Loureiro Queiroz, Marcos Homrich Hickmann, Indra Rojas Solano, Mauricio Alexandre Kuntzler e Manuel Martin Pino, mais Shaun Schottmiller.

2 Traduzido por Dayane Conceição da Silva de UFRGS. Revisado por Antonia Espíndola Longoni Klee. Agradece também a Lisianne Cabral de Melo, e Ruy Borboremo Neto, mestrado de UFPA. 
A verdade singular, apesar de alguma perspectiva ou julgamento sobre as consequiências de tal fato, é que a atividade humana está afetando o meio ambiente natural do nosso planeta. O que nós, como uma sociedade global ou comunidade internacional, escolhemos fazer sobre isso é o desafio para o futuro do $D I A$. Nesse sentido, o DIA deve, por necessidade, envolver todos os interessados, explorar a perspicácia, o discernimento, a penetração, a introspeç̧ão e o conhecimento de todas as culturas e disciplinas acadêmicas, permanecer "translimitada" em cada aspecto - em relação a barreiras políticas, de uma espécie ou ecossistema para outro - integrando todos os aspectos da natureza, formas de vida e recursos naturais. Os juízes, promotores, procuradores, aplicadores da lei em geral?) e estudantes de Direito podem contribuir formando uma lógica especial e uma disciplina da ciência dọ Direito sobre esses desafios globais, trazendo rigor e visão para a elaboração de acordos e declarações internacionais e criando meios de usar a linguagem cuidadosamente e precisamente para motivar a consideração das consequuências de qualquer atividade humana realizada.

Se as pessoas decidirem proceder com a atividade proposta, ou abster-se dela, o trabalho dos aplicadores do direito) terá sido esclarecer as escolhas e suas implicações. No entanto, precisamos ir ao encontro de muitas disciplinas além do Direito e analisar o trabalho dos ordenamentos legais internacionais representando muitas culturas, sistemas legais e prioridades políticas, tais como a Comissão do Direito Internacional da ONU e os grupos legais especializados em DIA, como a União Global da Conservação da Natureza ou os congressos da Associação Internacional do Direito, mais no Brasil, o trabalho do Instituto do Direito por um Planeta Verde.

A rica literatura em DIA de sábios de mundo todo está crescendo e é particularmente enriquecida pelas contribuições dos aplicadores do Direito) em variados sistemas legais e regiões do mundo, onde os recursos naturais são mais abundantes - como é o caso da maior biodiversidade encontrada em Estados equatoriais - ou onde as escolhas políticas são mais imperativas - como é o caso em que a rápida industrialização ou uma possível reivindicação do consumidor por produtos - está ameaçando imaculadas florestas ou recursos aquáticos com uso abusivo ou poluição. A bibliografia que segue consiste principalmente de fontes norte-americanas e brasileiras.

\section{OBJETIVOS}

Chegar a um entendimento da história e dos recursos do DIA e das instituições internacionais em que os conceitos são desenvolvidos e aplicados, assim como os princípios básicos do DIA, incluindo o princípio de precaução, eqüidade inter- e intra- geracional, a obrigação de não causar danos a outros e sua relação com o princípio de soberania sobre os recursos naturais. As preocupações especiais de países desenvolvidos e em desenvolvimento serão consideradas em respeito ao objetivo do desenvolvimento sustentável. 


\section{METODOLOGIA}

A metodologia adotada será aquela segundo a qual uma análise rigorosa do direito positivo no campo do DIA será seguida pela discussão do grupo, pesquisa e apresentações feitas por cada estudante na sala de um trabalho escrito. Questões sobre o possível ou desejável balanço de interesses serão levantadas. Quando adequado, estudos casuais de problemas atuais no $D I A$ e exemplos comparativos de jurisprudência nacional serão examinados. A elaboração de exercícios pode ser útil para conhecer a dificuldade enfrentada por advogados internacionais para escolher as palavras exatas para acordos e declarações, levando em consideração desafios particulares de diferença cultural, preferências lingüísticas exigências políticas e informação científica (ou falta de). Enfoques regionais, assim como programas e documentos globais serão analisados por suas receptividades para desafios de recursos naturais específicos dentro de uma situação local específica, relacionando essa análise com os princípios básicos do $D I A$ e acordos internacionais ou soft lawe.

\section{TEMAS ESPECÍFICOS}

1. Introdução Geral e História do Direito Internacional Ambiental (DIA)

2. Princípios de Direito Internacional Ambiental (DIA):

(Princípio de Precaução e Prevenção, Eqüidade, Obrigação de Não Causar Dano aos Outros)

3. A Relação entre os Princípios do DIA

4. As Instituições Internacionais e e DIA

5. Participação dos Atores/ "Stakeholders" no DIA

6. Jurisdição Internacional Ambiental

7. Direito Internacional Privado e Direito Internacional Público no Caso de DIA

8. Responsabilidade: Civil, Penal e Socio-ambiental

9. Solução dos Conflitos sobre DIA na ONU, OMC, CIJ

10. O Futuro do DIA: Um Direito de Desenvolvimento Sustentável?

\section{AVALIAÇÃO E CÁlulO: A IMPORTÂNCIA DOS GRUPOS DE DISCUSSÃ̃O, DA PESQUISA E DA APRESENTAÇÃO DE PROJETOS}

Os critérios de avaliação dependem do conhecimento do e domínio sobre o vasto corpo de acordos, doutrina e jurisprudência mundialmente. Além disso, é valorizada a habilidade de imaginar novos conceitos ou técnicas para alcançar um objetivo, neste caso tido como sendo o desenvolvimento sustentável. Para este fim, com o reconhecimento de 
diferentes valores e interpretações sobre se o desenvolvimento é realmente sustentável e a que custo é apresentado à sociedade, esforços serão feitos para considerar todas as formas de aprendizado demonstrado, de discussões em sala de aula e participação em respostas a discussão sobre idéias oferecidas por outros alunos / participantes nas apresentações de grupos sobre temas específicos em sala de aula, assim como o trabalho final escrito por cada estudante. Em todas as tarefas, esforços serão feitos para estimular o diálogo e o respeito pela diferença.

\section{BIBLIOGRAFIA EM INGLÊS}

Livros no Biblioteca de ONU, Faculdade de Direito, UFRGS

A Bibliografía brasileira será oferecida em momento oportuno.

\section{COMPARATIVE LAW:}

1. Rudolph B. Schlesinger, Hans W. Baade, Peter E. Herzog, Edward M. Wise, Comparative Law: Cases.Text-Materials, $6^{\text {th }}$ ed., New York: Foundation Press, 1998, 1013 pp.

2. Vicki C. Jackson, Mark Tushnet, Comparative Constitutional Law, New York: Foundation Press, 1999, 1507 pp.

3. Mary Ann Glendon, Michael Wallace Gordon, Christopher Osakwe, Comparative Legal Traditions: Test, Materials and Cases, $2^{\text {nd }}$ ed., St. Paul, Minnesota: West Publishing, 1994, 772 pp.

4. John Henry Merryman, The Civil Law Tradition, An Introduction to the Legal Systems of Western Europe and Latin America, Stanford, California: Stanford University Press, 1969, $172 \mathrm{pp}$.

5. René David, French Law, Its Structure, Sources, and Methodology, translated by Michael Kindred, Baton Rouge: Louisiana State University Press, 1972, 222 pp.

6. Arthur Taylor von Mehren, James Russell Gordley, The Civil Law System, An Introduction to the Comparative Study of Law, $2^{\text {nd }}$ ed., Boston: Little, Brown, $1977,1243 \mathrm{pp}$.

7. Richard A. Danner, Marie-Louise H. Bernal, Introduction to Foreign Legal Systems, New York: Oceana Publications, 1994, 423 pp. (See especially Ch. 2, Daniel L. Wade, "Comparative Law: Academic Perspectives and Practical Legal Realities," pp. 15-45). 
8. John H. Barton, James Lowell Gibbs, Jr., Victor Hao Li, John Henry Merryman, Law in Radically Different Cultures, St. Paul, Minnesota: West Publishing, 1983, 960 pp. (See especially introductory notes, pp. 1-15).

9. Bermann, Goebel, Davey, Fox, Cases and Materials on European Union Law, $2^{\text {nd }}$ ed., St. Paul, Minnesota: West Publishing, 2002, 1434 pp.

10. Document Supplement to Bermann, Goebel, Davey, Fox.

\section{INTERNATIONAL LAW AND INTERNATIONAL ENVIRONMENTAI} LAW:

1. Oscar Schachter, International Law in Theory and Practice, Martinus Nijhoff, 1991). $431 \mathrm{p}$.

2. Rosalyn Higgins, Problems and Process: International Law and How We Use It (Oxford: Clarendon Press, 1994). 274 p.

3. Ian Brownlie, Principles of Public International Law, $4^{\text {th }}$ ed. (Oxford: Clarendon Press, 1990). 748 p.

4. Edith Brown Weiss, In Fairness to Future Generations (Dobbs Ferry, NY: Transnational, 1989). $385 \mathrm{p}$.

5. Thomas Franck, Fairness in International Law and Institutions (Oxford: Clarendon Press, 1995). $500 \mathrm{p}$.

6. David Bederman, International Law Frameworks (New York: Foundation Press, 2001). 284 p.

7. Michael Akehurst, $A$ Modern Introduction to International Law, $6^{\text {th }}$ ed. (London: Allen and Unwin, 1987). 315 p.

8. Mark W. Janis, An Introduction to International Law, $2^{\text {nd }}$ ed. (Boston: Little, Brown, 1993).

9. Lea Brilmayer, Justifuying Interational Acts. (Ithaca, NY: Cornell University Press, 1989).

10. Gunther Handl, Yearbook of International Environmental Law (Boston: Graham and Trotman, 1991).

11. Andrew Hurrell and Benedict Kingsbury, eds., The International Politics of the Environment (Oxford: Clarendon Press, 1992).

12. Alexandre Kiss and Dinah Shelton, Manual of European Environmental Law. (Cambridge: Grotius Publications, 1993). 525 pp. 
13. Peter Wetterstein, ed., Harm to the Environment: The Right to Compensation and the Assessment of Damages (Oxford: Clarendon Press, 1997). 263 p.

14. Patricia Thomas, ed., Environmental Liability (London, Dord recht, Boston: Graham \& Trotman and International Bar Association, 1991), 399 p.

15. Practitioner's Deskbook Series: The Law of the WTO: Final Text of the GATT Uruguay Round Agreements, Summary, E Diskette. (New York: Oceana, 2002).

16. Sir Arthur Watts, International Law and the Antarctic Treaty System. (Cambridge: Grotius, 1992). 469 p.

\section{KLUWER: Graham \& Trotman, Martinus Nijhoff}

\section{International Environmental Law and Policy Series:}

17. Stanley P. Johnson, Guy Corcelle. The Environmental Policy of the European Communities. (London, Dordrecht, Boston: Kluwer, 1989). 349 p.

18. Harold Hohmann. Precautionary Legal Duties and Principles of Modern International Environmental Law (London, Dordrecht, Boston: Kluwer, 1994), $377 \mathrm{p}$.

19. Gunther Handl and Robert E. Lutz. Transferring Hazardous Tecbnologies and Substances. (London, Dordrecht, Boston: Kluwer, 1989). 275 p.

20. Francesco Francioni, Tullio Scovazzi, eds. International Responsibility for Environmental Harm (London, Dordrecht, Boston: Kluwer, 1991), 499 p.

21. Winfried Lang, Hanspeter Neuhold, Karl Zemanek, eds. Environmental Protection and International Law (London, Dordrecht, Boston: Kluwer, 1991), $244 \mathrm{p}$.

22. Turner T. Smith and Pascale Kromarek, eds. Understanding US and European Environmental Law: A Practitioner's Guide. (London, Dordrecht, Boston: Kluwer, 1989). $176 \mathrm{p}$.

23. Robin Churchill and David Freestone. International Law and Global Climate Change. (London, Dordrecht, Boston: Kluwer, 1991). 447 p.

24. Joe Verhoeven, Philippe Sands, Maxwell Bruce, eds. The Antarctic Environment and International Law. (London, Dordrecht, Boston: Kluwer, 1992). $228 \mathrm{p}$. 


\section{CASEBOOKS:}

25. Damrosch, Henkin, Pugh, Schachter, Smit, International Law, $4^{\text {th }}$ ed. (St. Paul, Minnesota: West Publishing, 2001), 1678 p.

26. Document Supplement to Damrosch, Henkin, Pugh, Schachter, Smit.

27. Weston, Falk and Charlesworth, International Law and World Order, $3^{\text {rd }}$ ed. (St. Paul, Minnesota: West Publishing, 1997), 1441 p.

28. Document Supplement to Weston, Falk and Charlesworth.

29. Guruswamy, Palmer, Weston, Carlson, International Environmental Law and World Order, $2^{\text {nd }}$ ed. (St. Paul, Minnesota: West Publishing, 1999), 1297 p.

30. Document Supplement to Guruswamy, Palmer, Weston, Carlson.

31. Hunter, Salzman, Zaelke, International Environmental Law and Policy, $2^{\text {nd }}$ ed. (New York: Foundation Press, 2002), 1547 p.

32. Document Supplement to Hunter, Salzman, Zaelke.

33. Document Supplement to Jackson, Davey, Sykes, Legal Problems of International Economic Relations. $3^{\text {rd }}$ Ed. (Minnesota: West Publishing, 1995).

34. Kirgis, International Organizations in Their Legal Setting, $2^{\text {nd }}$ ed.. (Minnesota: West Publishing, 1993). 1119 p.

35. Document Supplement to Kirgis

\section{INTERNATIONAL RELATIONS:}

1. Robert L. Rothstein, ed., The Evolution of Theory in International Relations, Columbia, S.C.: University of South Carolina Press, 1991, 222 p. (see especially Kenneth N. Waltz, "Realist Thought and Neo-Realist Theory," pp. 21-37; and Louis Henkin, "Law and Politics in International Relations: State and Human Values," pp. 163-188).

2. Donald M. Snow, Cases in International Relations, New York: Longman, 2003, 322 p. (see especially Ch. 2, "The Growing Significance of NGOs: Medecins sans Frontieres and Peacekeeping," pp. 21-36; Ch. 5, "Sovereignty and the Right of Intervention in Internal Wars: A Study of the Assault on State Sovereignty," pp. 77-93; Ch. 13, "The Milenium Summit: Rhetoric or Agenda of the 2000s," pp. 235-252; Ch. 14, "Let Them Drink Oil': Resource Conflict in the New Century," pp. 253-271). 
3. Smith, Booth and Zalewski, eds., International Theory: Positivism and Beyond, Cambridge: Cambridge University Press, 1996, 362 pp. (See especially Steve Smith, "Positivism and Beyond," pp. 11-46; Richard Little, "The Growing Relevance of Pluralism," pp. 66-86; Christine Sylvester, "The Contributions of Feminist Theory to International Relations," pp. 254-278; Andrew Linklater, "The Achievements of Critical Theory," pp. 279-300; James Rosenau, "Probing Puzzles Persistently: A Desirable but Improbable Future for IR Theory," pp. 309-317.

4. R.B.J. Walker, Inside-Outside: International Relations as Political Theory, Cambridge: Cambridge University Press, 1993, 233 p. (See especially Ch. 7, "Cosmopolitan Charm and Cultivated Cynicism, and Democracy and Political Community," pp. 141-152; and Ch. 8, "Sovereignty, Identity, Difference, and Rearticulations of Political Space-time," pp. 176-183.

5. Paul F. Diehl, ed., The Politics of International Organizations: Patterns and Insights, Chicago: The Dorsey Press, 1989. 468 pp. (See especially Oran Young, "International Regimes: Problems of Concept Formation," pp. 28-50; Susan Strange, "Cave bic dragones: A Critique of Regime Analysis," pp. 51-65; Robert Cox and Harold Jacobson, "The Framework for Inquiry," pp. 101-116; Craig Murphy, "What the Third World Wants: An Interpretation of the Development and Meaning of the New International Economic Order Ideology," pp. 226-241).

6. Jean-Marc Coicaud and Veijo Heiskanen, eds., The Legitimacy of International Organizations, New York: United Nations University Press, 2001, 578 pp. (See especially Jose Alvarez, "Constitutional Interpretation in International Organizations," pp. 104-154), Robert Howse, “The Legitimacy of the World Trade Organization," pp. 355-407).

7. Christopher Joyner, ed., The United Nations and International Law, Cambridge: Cambridge University Press, 1997, 474 pp.

8. Thomas G. Weiss and Leon Gordenker, eds., NGOs, the UN, E Global Governance, Boulder, Colorado: Lynne Rienner Publishers, 1996, 248 pp.

9. Cooper, English, Thakur, eds., Enhancing Global Governance: Towards A New Diplomacy, New York: United Nations University Press, 2002, 308 pp.

10. Crossing the Divide: Dialogue Among Civilizations, New Jersey: Seton Hall University, 2001, 252 pp.

11. Jürgen Habermas, The Structural Transformation of the Public Sphere, An Inquiry into a Category of Bourgeois Society, translated by Thomas Burger, Cambridge, Massachusetts: MIT Press, 1996, 301 pp. Original in German, Strukturwandel der Öffentlicheit, Darmstadt Neuwied, Germany: Hermann Luchterhand Verlag, 1962. 
12. David Harvey, The Condition of Postmodernity: An Enquiry into the Origins of Cultural Change, Cambridge, Massachusetts: Blackwell, 1990, 378 pp.

13. Saskia Sassen, Globalization and Its Discontents, Essays on the New Mobility of People and Money, New York: The New Press, 1998, 254 pp.

14. David Held and Anthony McGrew, eds., The Global Transformations Reader, An Introduction to the Globalization Debate, Cambridge, Massachusetts: Blackwell, 2000, 480 pp. (See especially Robert O. Keohane, "Sovereignty in International Society," pp. 109-123; Stephen D. Krasner, "Compromising Westphalia," pp. 124-135; David Held, "International Law," pp. 167-171; Onora O’Neill, "Transnational Justice," pp. 442-452).

15. Making Global Trade Work for People, London: Earthscan Publications, 2003, 341 pp. (See especially Ch. 3, "Towards a Human Development-Oriented Global Trade Regime," pp. 63-72; Ch. 4, "Reforms to the Global Governance of Trade," pp. 73-104; Ch. 18, "Trade and Environmental Policy," pp. 317-333).

16. Thomas L. Friedman, Understanding Globalization: The Lexus and the Olive Tree, New York: Anchor Books, 2000, 490 pp.

17. Selected issues of the American Journal of International Law, 1997-2004.

18. Selected issues of Foreign Affairs, $2002 \& 2003$. 